\title{
Metabolic Profiling of Varronia curassavica Jacq. Terpenoids by Flow Modulated Two-Dimensional Gas Chromatography Coupled to Mass Spectrometry
}

\author{
Roselaine Facanali ${ }^{1}$, Marcia Ortiz Mayo Marques ${ }^{2}$ and Leandro Wang Hantao ${ }^{1, *(1)}$ \\ 1 Institute of Chemistry, University of Campinas, Campinas 13083-970, SP, Brazil; roselainefacanali@gmail.com \\ 2 Agronomic Institute, Campinas 13075-630, SP, Brazil; mortiz@iac.sp.gov.br \\ * Correspondence: wang@unicamp.br; Tel.: +55-019-3521-3083
}

Received: 15 February 2020; Accepted: 9 March 2020; Published: 13 March 2020

check for updates

\begin{abstract}
In this study, a metabolomic approach was used to investigate the effect of seasonality on the chemical composition and yield of anti-inflammatory active principle, $\alpha$-humulene, in the essential oil of three genotypes of Varronia curassavica Jacq. (Syn. Cordia verbenaceae). The essential oils were extracted by hydrodistillation and analyzed by comprehensive two-dimensional gas chromatography coupled to mass spectrometry (GC $\times$ GC-MS). The GC $\times$ GC approach a three-fold improvement in qualitative analysis (48 compounds were identified by GC-MS versus 135 by GC $\times$ GC-MS). The improved resolving power of GC $\times$ GC resolved important coelutions and enabled the detection of unusual substances in V. curassavica essential oil. The chromatographic data was analyzed by using peak table-based chemometrics, namely, principal component analysis (PCA) and hierarchical cluster analysis (HCA). The metabolic study showed that seasonality has a significant effect on the chemical composition. The $\alpha$-humulene content was affected by genotype and season. Spring and summer were the best harvest seasons for the yield of the active ingredient, found in higher concentrations in the VC2 genotype. The proposed metabolomic workflow was successfully applied to terpene analysis found in V. curassavica essential oil, and such results have broadened our understanding of the influence of seasonal factors on the specialized metabolism of the species.
\end{abstract}

Keywords: Varronia curassavica; essential oil; seasonality; gas chromatography (GC); comprehensive two-dimensional gas chromatography $(\mathrm{GC} \times \mathrm{GC})$; flow-modulated

\section{Introduction}

Essential oils (EOs) are sources of biologically active compounds that can be applied in food, agronomic, cosmetic, and pharmaceutical industries for the elaboration of perfumes, cosmetics, pharmaceuticals, and used as adjuvant in the formulation of medicines. An important example of this application is the essential oil of Varronia curassavica Jacq. (syn. Cordia verbenacea) - a Brazilian native species, which is mainly found in the Atlantic Rainforest biome, popularly known as 'erva-baleeira' [1].

$V$. curassavica is a medicinal and aromatic plant from the Boraginaceae family with significant economic importance. The EO produced from its leaves is used in the formulation of the topic anti-inflammatory medicine Acheflan $^{\circledR}$, a phytotherapeutic product fully developed with Brazilian technology and currently exported to Japan, Chile, Mexico, Costa Rica, Equator, Peru, the United States, and Canada [2,3]. In Brazil, the product is the leading prescription drug for chronic tendonitis and muscle pain, being responsible for more than $25 \%$ of the market sales [3]. The active principle responsible for anti-inflammatory activity of the EO is the sesquiterpene $\alpha$-humulene [4-6]. The substance $\alpha$-humulene is considered a chemical marker in the pharmaceutical industry for the quality control of V. curassavica essential oil. Besides its anti-inflammatory activity, the species has been demonstrated to 
have other medicinal properties, such as anti-allergenic, antiulcerogenic, antioxidant, antitumor [5,7-11], antibacterial, and antifungal [12,13].

The world's growing demand for natural products, including essential oils, especially from the pharmaceutical industry, depends on standardizing and elucidating the chemical profile of essential oils safely and effectively. Along with the genetic make-up, environmental factors, including seasonality can coordinate or modify the production rate and composition of essential oil [14-18], influencing EO effectiveness, safety, and stability.

Gas chromatography coupled to mass spectrometry (GC-MS) is the most frequently used hyphenated separation technique for the characterization and identification of volatile organic compounds found in such oils [19-22]. However, the complex nature of the samples and the existence of coelutions are a challenge for qualitative and quantitative analysis [23,24]. Even with high-resolution GC using narrow bore capillary columns, the separation of complex mixtures such as essential oils is still incomplete, with a high number of overlapping chromatographic peaks. [25,26].

In this context, comprehensive two-dimensional gas chromatography (GC $\times G C)$ is a well-established technique for the resolution of complex mixtures of volatile organic compounds. The enhanced resolving power of GC $\times \mathrm{GC}$ is attained by coupling two capillary columns interfaced by the modulator [27-31]. Among the available modulators, the cryogenic modulator is currently the most popular solution [32]. Flow modulators are an interesting alternative for GC $\times G C$ experiments, since their separation efficiency may be equivalent to those obtained by thermal modulators [32,33]. For instance, Cordero et al. [34] evaluated the potential of GC $\times$ GC with differential flow modulator in medium and high complexity essential oil samples. Mint, lavender and vetiver essential oils were used for this evaluation. The authors reported increased peak capacity attained by differential flow modulation for the analysis of EOs. Accordingly, the benefits of GC $\times$ GC have led to an ever-increasing application of the composite technique for the separation and identification of volatile organic compounds in many other fields [35-40], including metabolomics [41-43].

Metabolic profiling of plants is informative to gain an insight into the potential of natural products for commercial use. For instance, the composition of EO is impacted by important factors like geography (soil, altitude, and humidity), seasonality (temperature and light conditions), and genotype. Hence, the chemical composition must be somewhat constant and predictable for quality assurance of the final product for pharmaceutical purposes. All reports characterized individual samples of $V$. curassavica using 1D-GC-based methods [44,45]. The seasonal effect of essential oil composition was explored using GC-MS [46-48], but without taking into consideration the contribution of plant genotype [48,49]. Marques et al. [48] previously evaluated the seasonal variation of EO from $V$. curassavica Jacq. accessions for an entire year. However, the authors could not factor into the investigation the accurate contribution of the multiple genotypes, because the biological material was harvested from native plants found in different geographical locations. Furthermore, careful inspection of the 1D-GC chromatograms seemed to indicate significant coelutions, which may have omitted important information regarding EO composition. In this work, we investigated $V$. curassavica plants with three different genotypes commercially available. All plants were cultivated in the same green house to eliminate the interference of geographical conditions. In addition, a GC $\times$ GC-MS-based metabolomic approach was used for $V$. curassavica EO characterization to improve the chemical analysis, especially for terpenes with overlapping peaks and low-intensity peaks. More specifically, flow modulated comprehensive two-dimensional gas chromatography coupled with mass spectrometry (FM-GC $\times$ GC-MS) and chemometric tools were combined in order to interpret the metabolic profile. These results improved our understanding of the influence of seasonal factors on the specialized metabolism of the species and are important to establish reliable data regarding V. curassavica crop behavior to fully explore the potential of this plant for commercial use. Lastly, we have confirmed the benefits of using GC $\times$ GC for EO analysis by highlighting previously overlapped peaks in 1D-GC, which were detected for the first time by GC $\times$ GC. 


\section{Material and Methods}

\subsection{Plant Material}

Three genotypes of V. curassavica (VC1, VC2, and VC3) were evaluated, comprising a total of nine plants. The plants, obtained from commercial matrices and propagated by stables (VC1 and VC2) and tissue culture (VC3), were kept in 20-L plastic roofed pots at the Center of Plant Genetic Resources of the Agronomic Institute (IAC), located in the city of Campinas, State of São Paulo ( $22^{\circ} 52^{\prime}$ south latitude, $47^{\circ} 04^{\prime}$ west longitude, and $677 \mathrm{~m}$ altitude). The regional climate, according to the Köppen classification, is classified as Cwa-subtropical climate with hot summers and dry winters. The climatological data for the sampling period were obtained from the meteorological station of the Agrometric Information Center (Ciiagro) [50]. The aerial parts were harvested in winter (16 August 2018), spring (21 November 2018), summer (21 February 2019) and autumn (30 May 2019). The harvested leaves of $V$. curassavica were manually separated from the stems and dried at $40{ }^{\circ} \mathrm{C}$ in an air-circulation oven for $72 \mathrm{~h}$.

\subsection{Extraction of Essential Oil}

Essential oils were extracted by hydrodistillation in a Clevenger apparatus for a period of two hours. The essential oil was stored in a freezer $\left(-4^{\circ} \mathrm{C}\right)$ for further characterization of the chemical composition.

\subsection{Comprehensive Two-Dimensional Gas Chromatographic Analysis (GC×GC-FID/MS)}

Analyses of the essential oils from $V$. curassavica were performed on a GCXGC system, which comprised of a TRACE 1300 GC equipped with a flame ionization detector (FID) and an ISQ single transmission quadrupole mass spectrometer (QMS) (Thermo Scientific-Waltham, MA, USA). Differential flow modulation using the reverse fill/flush configuration was attained using INSIGHT modulator (SepSolve Analytical-Frankfurt, Germany). Data digitalization using FID was attained at $120 \mathrm{~Hz}$. A $40-450 \mathrm{~m} / \mathrm{z}$ scanning range were used producing $31 \mathrm{scans} \mathrm{s}^{-1}$. The transfer line and the ion source were operated at $300^{\circ} \mathrm{C}$ and $220^{\circ} \mathrm{C}$, respectively. Helium was used as carrier gas and auxiliary gas at constant flow rates of $0.5 \mathrm{~mL} \mathrm{~min}^{-1}$ and $20.0 \mathrm{~mL} \mathrm{~min}^{-1}$. The column set consisted of a primary $30 \mathrm{~m} \times 0.25 \mathrm{~mm}$-id $\times 0.25 \mu \mathrm{m}$ ( $\beta$ of 250 ) MEGA-5HT and a secondary $5 \mathrm{~m} \times 0.25 \mathrm{~mm}$-id $\times 0.25 \mu \mathrm{m}$ ( $\beta$ of 250) HP-50 + wall coated open tubular (WCOT) capillary columns (Agilent Technologies-Santa Clara, CA, USA). Deactivated fused silica capillaries were used as transfer lines for simultaneous MS $(5 \mathrm{~m} \times 0.18 \mathrm{~mm}$-id $)$ and FID $(5 \mathrm{~m} \times 0.32 \mathrm{~mm}$-id $)$ detection. This setup ensured a reproducible splitting of the ${ }^{2} \mathrm{D}$ effluent to FID (70\%) and MS (30\%). Sample introduction was performed using a split ratio of 1:20. The GC oven was programmed from 60 to $240{ }^{\circ} \mathrm{C}$ at $3{ }^{\circ} \mathrm{C} \mathrm{min}-1$. Modulation period was set to $5.0 \mathrm{~s}$ with a re-injection (flush) pulse of $100 \mathrm{~ms}$. The chromatographic analyses were performed in triplicate.

Xcalibur software (Thermo Scientific-Waltham, MA, USA) was used for data acquisition and processing was performed using GC Image software (Zoex-Houston, TX, USA). Identification of the terpenes was performed comparing the substance mass spectra with NIST database and filtered by the retention index (LTPRI) using the NIST WebBook and published literature [51]. Retention indices were determined by the injection of a homologous series of $C_{8}-C_{20} n$-alkanes (04070-1ML) (Merck-St. Louis, MO, USA) using the Van den Dool and Kratz equation [52]. The analytes were tentative identified through comparative analysis of mass spectra considering a minimum similarity correspondence of $80 \%$ and a deviation of \pm 25 LTPRI from the NIST and Adams [51].

\subsection{Data Analysis}

Analysis of variance (ANOVA) followed by Tukey test $(p<0.05)$ was applied to verify the differences between essential oil yields and $\alpha$-humulene contents. The results of the chemical analyses were submitted to multivariate statistical analyses, namely, principal components analysis (PCA), and hierarchical clustering analysis (HCA). Such models were built using the software XLSTAT-2020 
version (Addinsoft-Bordeaux, France). Heatmap were constructed using the on-line tool MetaboAnalist 4.0 [53].

\section{Results and Discussions}

\subsection{Essential Oil Content}

The average yield of the essential oils of the three genotypes harvested during the winter, spring, summer, and autumn seasons is shown in Table 1. The yield of essential oils varied from 0.22 to $0.60 \%$ $(v / w)$. The yields are in agreement with those reported in other studies, which showed values ranging from 0.16 to $2.74 \%$. [46-48].

Table 1. Average yield (\%) of the essential oil of the three genotypes of Varronia curassavica extracted by hydrodistillation. Distinct uppercase letters (A, B and AB) for the rows and lowercase (a) for columns represent statistically significant differences $(p<0.05)$.

\begin{tabular}{ccccc}
\hline \multirow{2}{*}{ Genotype } & \multicolumn{4}{c}{ Season } \\
\cline { 2 - 5 } & Winter $^{\text {B }}$ & Spring $^{\text {A }}$ & Summer $^{\text {AB }}$ & Autumn $^{\text {AB }}$ \\
\hline VC1 $^{\text {a }}$ & $0.22 \pm 0.04$ & $0.42 \pm 0.04$ & $0.32 \pm 0.08$ & $0.41 \pm 0.03$ \\
VC2 $^{\text {a }}$ & $0.36 \pm 0.06$ & $0.60 \pm 0.03$ & $0.46 \pm 0.02$ & $0.52 \pm 0.02$ \\
VC3 $^{\text {a }}$ & $0.29 \pm 0.04$ & $0.58 \pm 0.02$ & $0.45 \pm 0.03$ & $0.44 \pm 0.04$ \\
\hline
\end{tabular}

The analysis of variance (ANOVA) showed that there was a significant difference in the yield of essential oils $(p<0.05)$ depending on the season. Spring was the season with the highest production $(0.533 \%)$ and winter the season with the lowest production $(0.291 \%)$. Between summer and autumn there was no significant difference ( 0.407 and $0.397 \%$, respectively). The VC2 genotype showed the highest production of essential oil $(0.60 \pm 0.03 \%)$ in spring, a period that coincides with the flowering of the plant. Although this species blooms at any time of the year, it flowers with higher intensity during spring.

The correlation of the results presented in Table 1 with the meteorological data (Figure 1) allows us to ascertain that the climatic conditions also constitute a strong factor. The rainfall exhibited a significant variation, since the accumulated rainfall indexes that varied from 75 to $484 \mathrm{~mm}$. Matias and collaborators [46] also observed the influence of rainfall on the production of $V$. curassavica essential oil, which corroborates our findings.

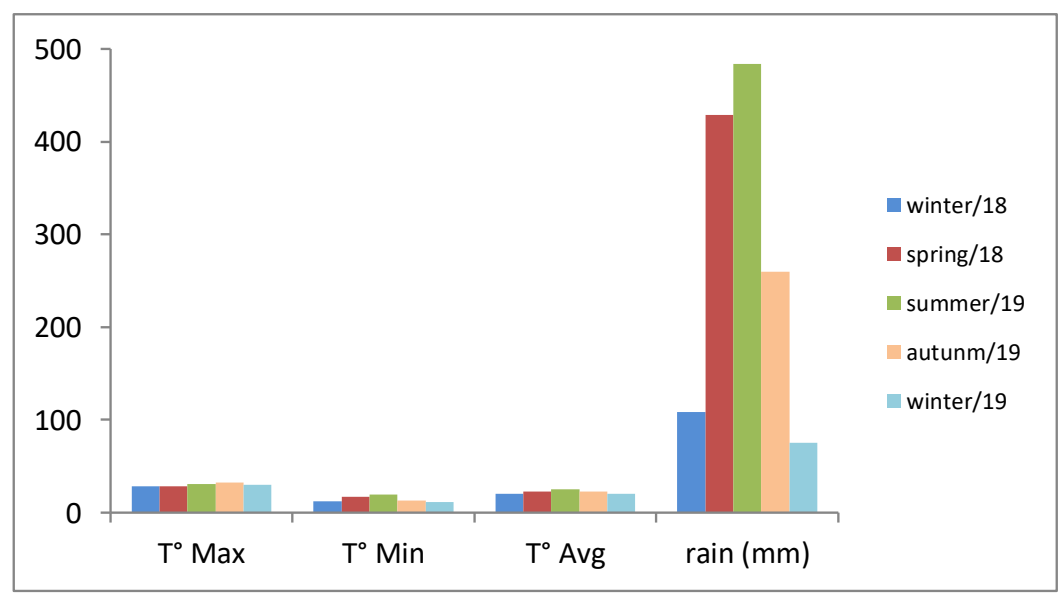

Figure 1. Meteorological data for the period of four seasons sampling. $\mathrm{T}^{\circ} \mathrm{Max}$-Maximum temperatures from July 2018 to July 2019; T Min-low temperatures from July 2018 to July 2019; T Avg-average temperature from July 2018 to July 2019; rain (mm)—pluviometric precipitation in the period July 2018 to July 2018. Fonte: CIIAGRO [50]. 


\subsection{Characterization of the Essential Oil}

The chemical compositions of the EOs of the three genotypes of V. curassavica, harvested in four different seasons, were analyzed by GC-MS and GCXGC-MS, as shown in Figure 2. Many substances that were not apparent in 1D-GC, due to overlapping peaks or because they are found in low intensities, are clearly revealed in the GC $\times$ GC chromatogram. An example of peaks that were co-eluted in the first dimension $\left({ }^{1} \mathrm{D}\right)$ and were resolved in the second dimension $\left({ }^{2} \mathrm{D}\right)$, as shown in Figure 3 . The composite system resolved the coelution between germacrene $\mathrm{D},(E)-\beta$-ionone and tau-elemene (Figure $3 \mathrm{~A}$ ) and between $\delta$-cadinene, cadin-1(2),4-diene, and two unidentified compounds (Figure 3B).
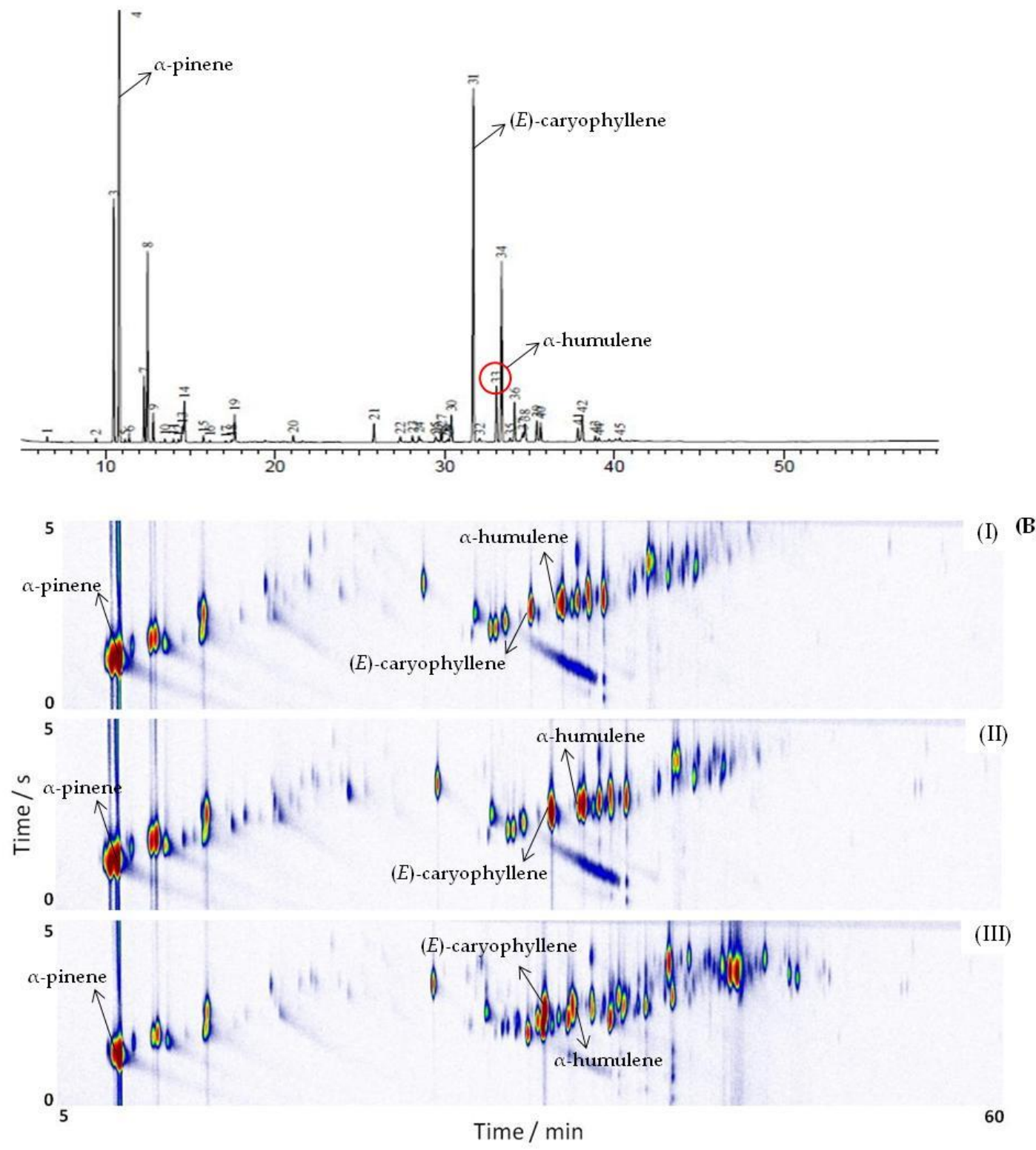

Figure 2. GC-MS chromatograms (A) and GC $\times$ GC-MS chromatograms (B) of the essential oil from three sample of V. curassavica (I) genotype VC1; (II) genotype VC2; (III) genotype VC3.

The GC $\times$ GC analysis enabled the tentative identification of 135 peaks compared to 48 peaks with GC-MS resulting in a three-fold improvement in qualitative analysis. Previous reports corroborated this improved feature for qualitative analysis [37,38,40,54]. For instance, Santos et al. [24] evaluated the essential oil of two species of Piperaceas (Manekia obtusa and Piper cubataonum). In M. obtuse oil, 80 compounds were identified by GC $\times$ GC-MS, while 22 only were assigned by GC-MS. For the leaf 
and branch oils of $P$. cubataonum, 57 and 66 compounds were identified by GC $\times$ GC compared with only 14 and 20 compounds by GC/MS, respectively.

It is important to note that although 168 compounds were detected in the GC $\times$ GC analysis, only $135(\sim 80 \%)$ were tentatively identified. The presence of very similar mass spectra imposed restraints to fully elucidate the compounds present in the EO.

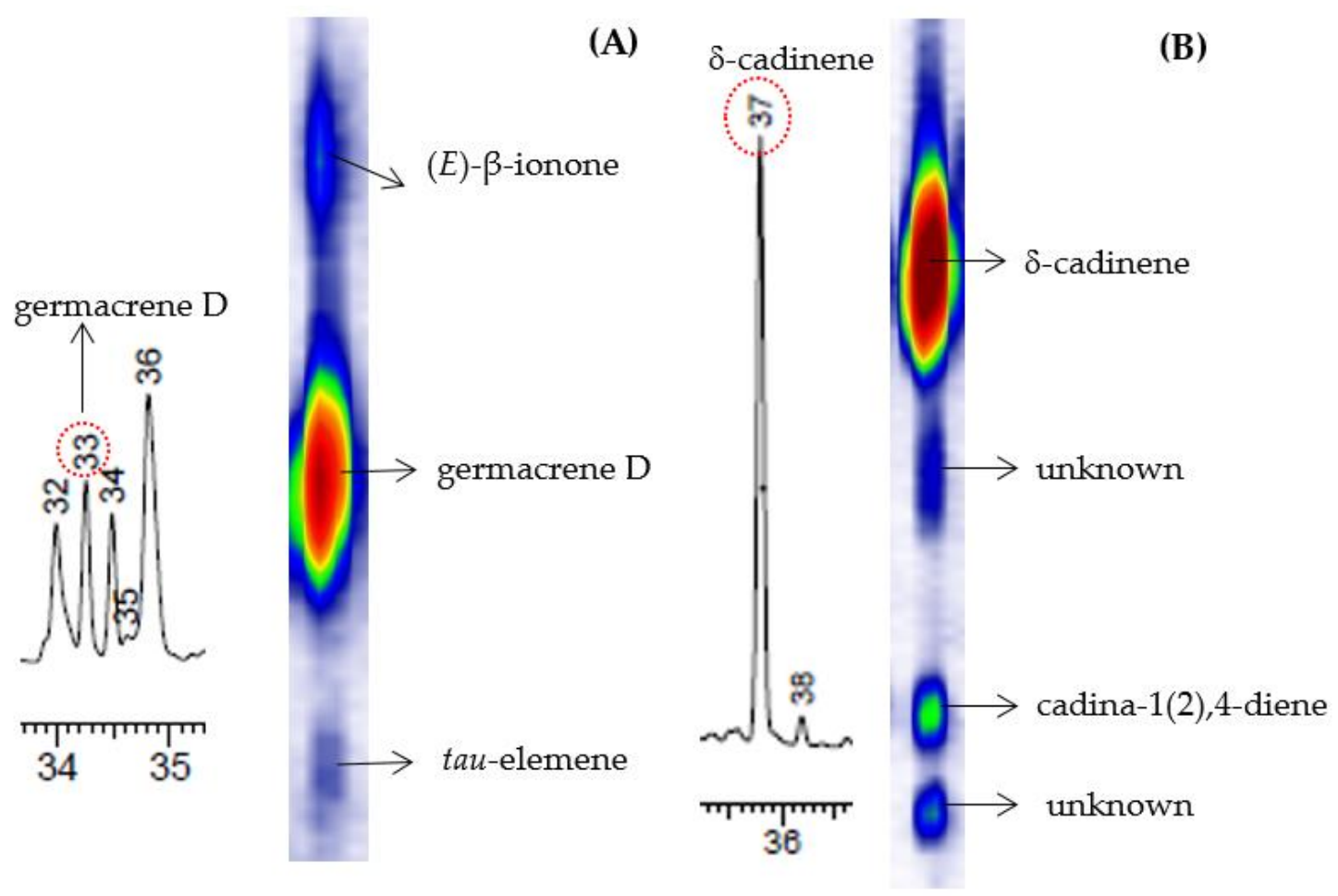

Figure 3. Example of improved peak capacity illustrating the resolution of two overlapping clusters (\#33 and \#37) in the first dimension of the chromatograms for essential oil V. curassavica. GC $\times$ GC resolved germacrene $\mathrm{D}(\mathbf{A})$ and $\delta$-cadinene $(\mathbf{B})$ as shown in the contour plots. The identified compounds and their average relative proportions are shown in Table 2 . The chemical classes of the analytes found in the EO of V. curassavica were monoterpenes and sesquiterpenes ( $96 \%$ of the oil).

The analysis of essential oils by GCXGC-FID/MS showed that $\alpha$-pinene (27.1-58.9\%) and (E)-caryophyllene (7.1-23.9\%) were the terpenes with higher relative proportions in all $\mathrm{EO}$ of the three genotypes, regardless of the harvesting season. However, $\alpha$-pinene and $(E)$-caryophyllene exhibited different relative proportions across all genotypes. Other terpenes were also detected in high intensities, such as $\alpha$-tujene, sabinene, $\beta$-pinene, 1,8-cineol, and germacrene D. Noteworthy, allo-aromadendrene, and spathulenol were found only in the VC1 and VC2 genotypes. Terpenes like $\alpha$-santalene, (E)- $\alpha$-santalal, and (E)- $\alpha$-bergamotenal were detected only in the VC3 genotype (Table 2).

Among the identified compounds, neryl acetate was detected only in genotypes VC2 and VC3 in the winter samples. $\beta$-copaene was detected in genotype VC1 (winter and autumn) and genotype VC2 (summer only). Terpene cis-muurol-5-en-4-ol was detected only in genotype VC2 (winter and spring), while cis-sesquisabinene hydrate was found only in VC3 (winter and spring). This variation in EO composition with seasonality is supported by previous reports [55,56]. Furthermore, different genotypes cultivated under the same conditions may exhibit differential expressions resulting in distinct chemical profiles, including the occurrence or absence of metabolites [57]. 
Table 2. Chemical composition (relative \%) of Varronia curassavica essential oils harvested in different seasons and analyzed by GC $\times$ GC-FID/MS. LTPRI - Linear temperature programmed retention indices; Exp.-LTPRI experimental obtained by the injection of a homologous series of C8-C20 n-alkanes using the Van den Dool and Kratz equation [52]; Lit. - LTPRI obtained from literature [51] and NIST WebBook. $\left({ }^{*}\right)$-LTPRI not found; $\left({ }^{* *}\right)$-Substance identified by comparison with Sciarrone and collaborators [44]; (ta) - Trace amounts $(\leq 0.003) ;(-)$ - Absence of the metabolite.

\begin{tabular}{|c|c|c|c|c|c|c|c|c|c|c|c|c|c|c|}
\hline \multirow{3}{*}{ Substance } & \multirow{2}{*}{\multicolumn{2}{|c|}{ LTPRI }} & \multicolumn{12}{|c|}{ Genotype/Season } \\
\hline & & & \multicolumn{4}{|c|}{ VC1 } & \multicolumn{4}{|c|}{ VC2 } & \multicolumn{4}{|c|}{ VC3 } \\
\hline & Exp. & Lit. & Winter & Spring & Summer & Autumn & Winter & Spring & Summer & Autumn & Winter & Spring & Summer & Autumn \\
\hline tricyclene & 930 & 926 & 0.464 & ta & ta & ta & 1.074 & ta & ta & ta & 0.091 & ta & ta & ta \\
\hline$\alpha$-thujene & 939 & 930 & 8.046 & 5.929 & 6.047 & 9.273 & 9.369 & 7.188 & 7.154 & 9.392 & 7.479 & ta & 0.032 & 0.078 \\
\hline$\alpha$-pinene & 947 & 939 & 28.920 & 35.236 & 33.769 & 48.178 & 27.102 & 29.058 & 33.985 & 33.062 & 33.029 & 41.899 & 45.805 & 58.864 \\
\hline thuja-2,4(10)-diene & 956 & 951 & ta & ta & 0.036 & ta & 0.026 & ta & 0.067 & ta & - & - & - & - \\
\hline camphene & 963 & 954 & 0.233 & 0.212 & 0.161 & 0.142 & 0.288 & 0.252 & 0.181 & 0.182 & 0.245 & 0.172 & 0.158 & 0.133 \\
\hline sabinene & 983 & 975 & 2.048 & 1.353 & 1.401 & 1.621 & 2.557 & 1.387 & 1.321 & 2.333 & 0.614 & 0.372 & 0.414 & 0.305 \\
\hline$\beta$-pinene & 990 & 979 & 3.179 & 2.734 & 2.535 & 3.178 & 5.997 & 5.341 & 4.898 & 5.702 & 2.106 & 1.530 & 1.517 & 1.585 \\
\hline myrcene & 998 & 990 & 0.547 & 0.320 & 0.403 & 0.367 & 0.829 & 0.427 & 0.359 & 0.647 & 0.442 & 0.113 & 0.296 & 0.212 \\
\hline$\alpha$-phellandrene & 1019 & 1002 & 0.038 & 0.079 & 0.079 & 0.124 & 0.088 & 0.079 & 0.074 & 0.128 & 0.027 & 0.019 & 0.043 & ta \\
\hline$\alpha$-terpinene & 1027 & 1017 & ta & ta & 0.036 & ta & 0.031 & ta & 0.053 & 0.051 & - & - & - & - \\
\hline o-cymene & 1037 & 1022 & 0.122 & ta & 0.044 & ta & 0.123 & ta & 0.049 & ta & 0.026 & ta & 0.006 & ta \\
\hline limonene & 1024 & 1024 & ta & ta & ta & 0.147 & ta & ta & ta & 0.147 & - & - & - & - \\
\hline$\beta$-phellandrene & 1029 & 1025 & ta & 0.043 & ta & ta & ta & 0.030 & ta & ta & ta & 0.532 & ta & ta \\
\hline sylvestrene & 1038 & 1025 & 0.492 & 0.556 & 0.590 & 0.310 & 0.356 & 0.367 & 0.392 & 0.349 & 0.635 & ta & 0.721 & 0.399 \\
\hline 1,8-cineole & 1041 & 1026 & 2.016 & 1.412 & 1.019 & 1.455 & 2.269 & 1.112 & 0.702 & 1.855 & 1.039 & 0.853 & 0.854 & 0.600 \\
\hline (E)- $\beta$-ocimene & 1044 & 1049 & ta & ta & 0.015 & ta & ta & ta & 0.025 & ta & ta & ta & 0.025 & ta \\
\hline$\gamma$-terpinene & 1070 & 1059 & 0.040 & 0.078 & 0.107 & 0.085 & 0.100 & 0.076 & 0.154 & 0.077 & 0.021 & ta & 0.023 & ta \\
\hline cis-sabinene hydrate & 1081 & 1070 & 0.069 & 0.043 & 0.026 & 0.030 & 0.119 & 0.034 & 0.042 & ta & 0.018 & ta & 0.012 & ta \\
\hline terpinolene & 1102 & 1088 & ta & ta & 0.014 & ta & ta & 0.019 & 0.026 & ta & - & - & - & - \\
\hline linalool & 1108 & 1096 & 0.057 & ta & ta & 0.052 & 0.031 & ta & ta & ta & - & - & - & - \\
\hline$\alpha$-pinene oxide & 1108 & 1097 & 0.078 & ta & ta & ta & 0.021 & ta & ta & ta & 0.105 & $\operatorname{tr}$ & $\operatorname{tr}$ & $\operatorname{tr}$ \\
\hline trans-sabinene hydrate & 1111 & 1098 & 0.029 & 0.034 & ta & ta & 0.108 & 0.034 & 0.027 & ta & 0.009 & $\operatorname{tr}$ & $\operatorname{tr}$ & $\operatorname{tr}$ \\
\hline n-nonanal & 1115 & 1110 & 0.444 & ta & 0.243 & ta & 0.344 & ta & 0.181 & ta & 0.119 & $\operatorname{tr}$ & 0.095 & $\operatorname{tr}$ \\
\hline (E)-3(10)-caren-4-ol & 1130 & $*$ & 0.018 & ta & ta & ta & 0.043 & ta & ta & ta & - & - & - & - \\
\hline cis-p-menth-2-en-1-ol & 1134 & 1124 & - & - & - & - & 0.023 & ta & ta & ta & - & - & - & - \\
\hline$\alpha$-campholenal & 1126 & 1139 & ta & ta & 0.033 & ta & 0.025 & ta & 0.028 & ta & 0.015 & 0.012 & 0.018 & $\operatorname{tr}$ \\
\hline trans-pinocarveol & 1152 & 1139 & 0.036 & ta & 0.051 & ta & ta & ta & 0.041 & ta & 0.028 & $\operatorname{tr}$ & 0.019 & $\operatorname{tr}$ \\
\hline isopinocarveol & 1151 & 1140 & ta & 0.034 & 0.016 & ta & ta & 0.026 & 0.019 & ta & $\operatorname{tr}$ & 0.032 & 0.019 & $\operatorname{tr}$ \\
\hline trans-sabinol & 1157 & 1142 & 0.061 & 0.017 & ta & ta & 0.049 & ta & 0.036 & ta & 0.034 & $\operatorname{tr}$ & $\operatorname{tr}$ & $\operatorname{tr}$ \\
\hline camphor & 1157 & 1146 & 0.029 & 0.034 & 0.028 & ta & - & - & - & - & - & - & - & - \\
\hline
\end{tabular}


Table 2. Cont

\begin{tabular}{|c|c|c|c|c|c|c|c|c|c|c|c|c|c|c|}
\hline \multirow{3}{*}{ Substance } & \multirow{2}{*}{\multicolumn{2}{|c|}{ LTPRI }} & \multicolumn{12}{|c|}{ Genotype/Season } \\
\hline & & & \multicolumn{4}{|c|}{ VC1 } & \multicolumn{4}{|c|}{ VC2 } & \multicolumn{4}{|c|}{ VC3 } \\
\hline & Exp. & Lit. & Winter & Spring & Summer & Autumn & Winter & Spring & Summer & Autumn & Winter & Spring & Summer & Autumn \\
\hline pinocarvone & 1174 & 1164 & 0.032 & ta & 0.039 & ta & 0.030 & ta & ta & ta & 0.015 & 0.009 & 0.011 & $\operatorname{tr}$ \\
\hline borneol & 1182 & 1169 & ta & ta & 0.009 & ta & ta & 0.012 & ta & ta & 0.010 & 0.015 & 0.008 & $\operatorname{tr}$ \\
\hline terpinen-4-ol & 1190 & 1177 & 0.105 & 0.108 & 0.103 & 0.074 & 0.166 & 0.077 & 0.180 & 0.112 & 0.033 & 0.017 & 0.022 & $\operatorname{tr}$ \\
\hline$\alpha$-terpineol & 1209 & 1188 & 0.033 & ta & 0.016 & ta & 0.034 & ta & 0.022 & ta & 0.006 & $\operatorname{tr}$ & $\operatorname{tr}$ & $\operatorname{tr}$ \\
\hline$\beta$-cyclocitral & 1229 & 1219 & ta & ta & 0.018 & ta & ta & ta & ta & ta & 0.007 & $\operatorname{tr}$ & $\operatorname{tr}$ & $\operatorname{tr}$ \\
\hline citronellol & 1223 & 1229 & ta & ta & 0.012 & ta & ta & ta & 0.017 & ta & - & - & - & - \\
\hline bornyl acetate & 1290 & 1285 & 0.857 & 0.466 & 0.451 & 0.373 & 1.096 & 0.720 & 0.830 & 0.633 & 0.706 & 0.557 & 0.671 & 0.537 \\
\hline trans-pinocarvyl acetate & 1302 & 1298 & - & - & - & - & - & - & - & - & 0.005 & 0.014 & $\operatorname{tr}$ & $\operatorname{tr}$ \\
\hline myrtenyl acetate & 1329 & 1326 & - & - & - & - & - & - & - & - & 0.013 & $\operatorname{tr}$ & 0.009 & $\operatorname{tr}$ \\
\hline 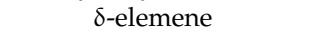 & 1338 & 1338 & 0.010 & 0.022 & 0.019 & 0.057 & 0.006 & 0.024 & 0.012 & ta & - & - & - & - \\
\hline$\alpha$-cubebene & 1351 & 1351 & 0.061 & 0.069 & 0.064 & 0.052 & 0.037 & 0.042 & 0.034 & ta & 0.005 & 0.017 & $\operatorname{tr}$ & $\operatorname{tr}$ \\
\hline citronellyl acetate & 1353 & 1352 & 0.592 & 0.178 & 0.143 & 0.159 & 0.434 & 0.098 & 0.113 & 0.222 & 0.315 & 0.087 & 0.217 & 0.069 \\
\hline neryl acetate & 1361 & 1365 & - & - & - & - & 0.008 & - & - & - & 0.011 & - & - & - \\
\hline cyclosativene & 1373 & 1371 & 0.909 & 0.669 & ta & 0.420 & ta & 0.472 & ta & 0.356 & 0.072 & 0.061 & ta & ta \\
\hline$\alpha$-Ylangene & 1375 & 1373 & ta & ta & 0.511 & ta & 0.566 & ta & 0.272 & ta & ta & ta & 0.035 & ta \\
\hline$\alpha$-copaene & 1380 & 1376 & 1.568 & 1.340 & 1.278 & 0.879 & 0.716 & 0.671 & 0.537 & 0.417 & 0.079 & 0.078 & 0.037 & 0.042 \\
\hline$\beta$-bourbonene & 1387 & 1388 & 0.069 & 0.193 & 0.212 & 0.125 & 0.127 & 0.117 & 0.148 & 0.081 & 0.006 & 0.014 & ta & ta \\
\hline$\beta$-cubebene & 1393 & 1388 & 1.616 & 1.499 & 1.206 & 1.057 & 0.755 & 0.705 & 0.507 & 0.571 & - & - & - & - \\
\hline 7-epi-sesquithujene & 1389 & 1391 & - & - & - & - & - & - & - & - & 0.036 & 0.019 & 0.051 & 0.022 \\
\hline$\beta$-longipinene & 1410 & 1403 & 0.033 & 0.032 & ta & 0.060 & 0.041 & 0.034 & ta & ta & - & - & - & - \\
\hline sesquithujene & 1404 & 1405 & - & - & - & - & - & - & - & - & 1.438 & 1.473 & 1.192 & 1.027 \\
\hline (Z)-caryophyllene & 1410 & 1408 & - & - & - & - & - & - & - & - & 0.017 & 0.008 & 0.010 & ta \\
\hline cis- $\alpha$-bergamotene & 1418 & 1412 & - & - & - & - & - & - & - & - & 1.125 & 1.179 & 0.998 & 0.860 \\
\hline$\alpha$-santalene & 1425 & 1417 & - & - & - & - & - & - & - & - & 10.213 & 11.887 & 9.140 & 9.379 \\
\hline (E)-caryophyllene & 1427 & 1419 & 7.481 & 10.997 & 11.679 & 7.119 & 18.026 & 23.999 & 22.384 & 21.548 & 11.917 & 14.800 & 12.330 & 11.453 \\
\hline$\beta$-copaene & 1435 & 1432 & 0.111 & - & - & 0.051 & - & - & 0.076 & - & - & - & - & - \\
\hline trans- $\alpha$-bergamotene & 1435 & 1434 & - & - & - & - & - & - & - & - & 0.197 & 0.271 & 0.145 & 0.082 \\
\hline$\alpha$-guaiene & 1451 & 1439 & 0.024 & ta & 0.021 & ta & - & - & - & - & - & - & - & - \\
\hline aromadendrene & 1457 & 1441 & 0.020 & 0.172 & 0.070 & ta & 0.110 & 0.197 & 0.021 & ta & - & - & - & - \\
\hline (Z)- $\beta$-Farnesene & 1445 & 1442 & - & - & - & - & - & - & - & - & 0.212 & 0.207 & 0.171 & 0.105 \\
\hline epi- $\beta$-santalene & 1451 & 1447 & - & - & - & - & - & - & - & - & ta & 0.026 & 0.020 & 0.026 \\
\hline cis-muurola-3,5-diene & 1450 & 1449 & ta & ta & ta & ta & 0.010 & 0.033 & ta & ta & 0.035 & ta & ta & ta \\
\hline trans-muurola-3,5-diene & 1457 & 1453 & ta & 0.030 & ta & ta & ta & 0.034 & ta & ta & ta & ta & ta & ta \\
\hline sesquisabinene & 1454 & 1454 & - & - & - & - & - & - & - & - & ta & ta & 1.652 & ta \\
\hline$\alpha$-humulene & 1463 & 1454 & 1.470 & 2.383 & 2.442 & 1.378 & 2.649 & 3.821 & 3.934 & 3.284 & 2.892 & 3.456 & 2.880 & 1.574 \\
\hline (E)- $\beta$-farnesene & 1457 & 1456 & - & - & - & - & - & - & - & - & 1.862 & 1.835 & 0.057 & 1.004 \\
\hline allo-aromadendrene & 1469 & 1460 & 11.904 & 12.781 & 13.821 & 11.513 & 10.687 & 8.632 & 9.879 & 12.300 & - & - & - & - \\
\hline dehydro-aromadendrene & 1471 & 1462 & 0.541 & 0.312 & 0.573 & ta & 0.218 & ta & 0.276 & ta & 0.154 & 0.232 & ta & ta \\
\hline
\end{tabular}


Table 2. Cont

\begin{tabular}{|c|c|c|c|c|c|c|c|c|c|c|c|c|c|c|}
\hline \multirow{3}{*}{ Substance } & \multirow{2}{*}{\multicolumn{2}{|c|}{ LTPRI }} & \multicolumn{12}{|c|}{ Genotype/Season } \\
\hline & & & \multicolumn{4}{|c|}{ VC1 } & \multicolumn{4}{|c|}{ VC2 } & \multicolumn{4}{|c|}{ VC3 } \\
\hline & Exp. & Lit. & Winter & Spring & Summer & Autumn & Winter & Spring & Summer & Autumn & Winter & Spring & Summer & Autumn \\
\hline$\gamma$-muurolene & 1480 & 1479 & 0.840 & 0.589 & 0.532 & 0.290 & 0.256 & 0.251 & 0.168 & ta & 0.023 & 0.017 & ta & ta \\
\hline$\gamma$-elemene & 1480 & 1488 & ta & ta & 0.036 & 0.204 & - & - & - & - & - & - & - & - \\
\hline germacrene D & 1488 & 1481 & 1.716 & 2.965 & 3.879 & 1.585 & 0.015 & 3.548 & 4.855 & 2.066 & 0.592 & 0.498 & 1.170 & 0.383 \\
\hline tau-elemene & 1484 & 1484 & 0.383 & 0.539 & 0.216 & 0.139 & 0.175 & 0.163 & ta & ta & 0.264 & ta & ta & ta \\
\hline (E)- $\beta$-ionone & 1488 & 1488 & 0.168 & ta & ta & ta & 0.050 & ta & ta & ta & 0.059 & 0.244 & ta & ta \\
\hline cis- $\beta$-guaiene & 1492 & 1490 & ta & ta & 0.085 & 0.087 & ta & ta & 0.042 & ta & ta & ta & ta & ta \\
\hline$\alpha$-zingiberene & 1500 & 1493 & - & - & - & - & - & - & - & - & 0.020 & ta & 0.054 & 0.037 \\
\hline trans-muurola-4(14),5-diene & 1488 & 1493 & ta & ta & ta & ta & 2.062 & ta & ta & ta & ta & ta & 0.021 & ta \\
\hline$\gamma$-amorphene & 1496 & 1493 & ta & ta & ta & ta & 0.069 & ta & ta & 0.084 & ta & ta & ta & ta \\
\hline epi-cubebol & 1493 & 1494 & ta & ta & 0.698 & 0.121 & ta & ta & 0.160 & 0.124 & - & - & - & - \\
\hline biciclogermacrene & 1502 & 1500 & 1.283 & ta & 6.183 & 3.822 & 1.051 & ta & 2.166 & 1.690 & - & - & - & - \\
\hline$\alpha$-muurolene & 1504 & 1500 & 1.016 & ta & ta & ta & ta & ta & ta & 0.087 & 0.142 & ta & 0.057 & 0.037 \\
\hline trans- $\beta$-guaiene & 1504 & 1502 & 0.845 & ta & 0.022 & ta & 0.563 & ta & 0.017 & ta & - & - & - & - \\
\hline$\beta$-bisabolene & 1512 & 1505 & - & - & - & - & - & - & - & - & 2.498 & ta & 2.438 & 0.070 \\
\hline guaia-1(10),11-diene & 1516 & 1509 & 0.178 & 0.169 & ta & ta & 0.072 & 0.079 & ta & ta & - & - & - & - \\
\hline (Z)- $\gamma$-bisabolene & 1514 & 1514 & - & - & - & - & - & - & - & - & ta & ta & 0.274 & 0.034 \\
\hline cadina-1(2)4-diene & 1524 & 1519 & 0.023 & ta & ta & ta & 0.021 & ta & ta & ta & - & - & - & - \\
\hline cubebol & 1514 & 1521 & ta & ta & 1.186 & 1.107 & ta & ta & 0.552 & 0.317 & - & - & - & - \\
\hline$\beta$-sesquiphellandrene & 1521 & 1525 & - & - & - & - & - & - & - & - & ta & ta & 0.287 & 0.208 \\
\hline$\delta$-cadinene & 1524 & 1530 & 7.217 & 5.800 & 3.640 & 2.924 & 2.998 & 1.969 & 0.830 & 0.838 & 0.636 & ta & ta & ta \\
\hline (E)- $\gamma$-bisabolene & 1531 & 1531 & - & - & - & - & - & - & - & - & 1.002 & 1.390 & 0.886 & 0.487 \\
\hline trans-cadina-1,4-diene & 1541 & 1534 & 0.045 & 0.067 & 0.029 & 0.037 & 0.026 & 0.032 & 0.016 & ta & - & - & - & - \\
\hline (E)- $\alpha$-bisabolene & 1545 & 1545 & - & - & - & - & - & - & - & - & 0.023 & ta & 0.020 & 0.038 \\
\hline$\alpha$-calacorene & 1551 & 1545 & 0.045 & 0.020 & 0.018 & ta & - & - & - & - & - & - & - & - \\
\hline elemol & 1557 & 1549 & 0.035 & 0.047 & 0.017 & ta & - & - & - & - & - & - & - & - \\
\hline cis-muurol-5-en-4-ol & 1561 & 1551 & - & - & - & - & 0.048 & 0.047 & - & - & - & - & - & - \\
\hline cis-sesquisabinene hidrate & 1551 & 1559 & - & - & - & - & - & - & - & - & 0.110 & 0.053 & - & - \\
\hline germacrene $B$ & 1567 & 1561 & 0.277 & 0.185 & 0.063 & 0.083 & 0.133 & 0.086 & 0.036 & ta & - & - & - & - \\
\hline trans-sesquisabinene hydrate & 1561 & 1565 & - & - & - & - & - & - & - & - & 0.486 & 0.345 & ta & ta \\
\hline (E)-nerolidol & 1567 & 1565 & ta & ta & 0.037 & ta & ta & ta & ta & ta & 0.039 & ta & 0.039 & ta \\
\hline$\beta$-calacorene & 1573 & 1565 & 0.011 & ta & 0.004 & ta & ta & ta & ta & ta & ta & ta & ta & ta \\
\hline germacrene D-4-ol & 1586 & 1575 & ta & 0.059 & 0.057 & 0.041 & ta & 0.018 & 0.025 & ta & ta & ta & ta & ta \\
\hline spathulenol & 1588 & 1578 & 3.738 & 1.003 & 1.422 & 0.527 & 0.985 & 0.171 & 0.555 & 0.185 & - & - & - & - \\
\hline caryophyllene oxide & 1594 & 1583 & 0.726 & 0.424 & 0.438 & 0.198 & 2.524 & 0.502 & 0.636 & 0.274 & 2.509 & 1.031 & 0.901 & 0.644 \\
\hline 7-epi-trans-sesquisabinene hydrate & 1598 & $*$ & - & - & - & - & - & - & - & - & ta & ta & 0.629 & 0.327 \\
\hline salvial-4(14)-en-1-one & 1604 & 1594 & 0.122 & ta & ta & ta & ta & 0.015 & 0.021 & ta & ta & ta & ta & ta \\
\hline ledol & 1615 & 1602 & 0.716 & 0.437 & 0.410 & ta & 0.370 & 0.254 & 0.282 & ta & - & - & - & - \\
\hline
\end{tabular}


Table 2. Cont

\begin{tabular}{|c|c|c|c|c|c|c|c|c|c|c|c|c|c|c|}
\hline \multirow{3}{*}{ Substance } & \multirow{2}{*}{\multicolumn{2}{|c|}{ LTPRI }} & \multicolumn{12}{|c|}{ Genotype/Season } \\
\hline & & & \multicolumn{4}{|c|}{ VC1 } & \multicolumn{4}{|c|}{ VC2 } & \multicolumn{4}{|c|}{ VC3 } \\
\hline & Exp. & Lit. & Winter & Spring & Summer & Autumn & Winter & Spring & Summer & Autumn & Winter & Spring & Summer & Autumn \\
\hline humulene epoxide II & 1621 & 1608 & 0.349 & 0.067 & 0.083 & ta & 0.302 & 0.043 & 0.083 & ta & 0.284 & 0.075 & 0.049 & ta \\
\hline isoaromadendrene epoxide & 1612 & 1620 & ta & ta & ta & 0.237 & ta & ta & ta & 0.192 & - & - & - & - \\
\hline muurola-4,10(14)-dien-1-b-ol & 1636 & 1631 & 0.426 & 0.123 & 0.173 & 0.097 & - & - & - & - & - & - & - & - \\
\hline cis-Cadin-4-en-7-ol & 1636 & 1636 & ta & ta & ta & ta & 0.143 & 0.087 & 0.069 & 0.061 & ta & 0.017 & ta & ta \\
\hline isospathulenol & 1643 & 1639 & 0.263 & 0.162 & 0.100 & ta & 0.165 & 0.027 & 0.073 & ta & - & - & - & - \\
\hline caryophylla-4(12),8(13)-dien- $5 \alpha$-ol & 1649 & 1640 & ta & ta & ta & ta & 0.121 & ta & ta & ta & ta & 0.017 & ta & ta \\
\hline tau-cadinol & 1640 & 1640 & ta & ta & 0.045 & ta & ta & ta & 0.056 & 0.035 & ta & ta & ta & ta \\
\hline allo-aromadendrene epoxide & 1651 & 1641 & 0.054 & 0.108 & ta & ta & - & - & - & - & - & - & - & - \\
\hline cubenol & 1655 & 1646 & ta & ta & 0.015 & ta & ta & 0.064 & ta & ta & - & - & - & - \\
\hline$\alpha$-muurolol & 1657 & 1646 & 1.067 & 0.500 & 0.333 & 0.200 & 0.366 & 0.134 & 0.160 & 0.093 & 0.070 & 0.034 & ta & ta \\
\hline$\alpha$-cadinol & 1666 & 1654 & 0.113 & 0.081 & 0.151 & ta & 0.051 & 0.040 & 0.102 & ta & - & - & - & - \\
\hline cis-calamenen-10-ol & 1668 & 1661 & 0.300 & ta & ta & ta & ta & ta & ta & ta & ta & ta & ta & ta \\
\hline intermedeol & 1670 & 1666 & 0.123 & 0.070 & ta & ta & ta & 0.018 & ta & ta & ta & ta & ta & ta \\
\hline trans-10-hydroxycalamenene & 1679 & 1675 & 0.035 & ta & 0.025 & ta & - & - & - & - & - & - & - & - \\
\hline ledene oxide (II) & 1687 & 1682 & 0.093 & 0.045 & 0.045 & ta & - & - & - & - & - & - & - & - \\
\hline (2Z,6Z)-farnesal & 1684 & 1684 & ta & ta & 0.022 & ta & ta & ta & 0.008 & ta & ta & ta & ta & ta \\
\hline Z- $\alpha$-trans-bergamotol & 1668 & 1690 & - & - & - & - & - & - & - & - & 0.491 & 0.218 & ta & ta \\
\hline (E)- $\alpha$-bergamotenal & 1679 & $* *$ & - & - & - & - & - & - & - & - & 4.098 & 3.311 & 4.171 & 2.212 \\
\hline (E)- $\alpha$-Santalal & 1689 & $* *$ & - & - & - & - & - & - & - & - & 6.003 & 5.618 & 7.227 & 3.679 \\
\hline eudesm-7(11)-en-4-ol & 1707 & 1700 & 0.113 & 0.081 & ta & ta & 0.064 & 0.026 & ta & ta & - & - & - & - \\
\hline (Z)- $\beta$-santalol & 1716 & 1716 & - & - & - & - & - & - & - & - & 0.048 & 0.021 & 0.034 & ta \\
\hline$\beta$-santalol & 1723 & 1727 & - & - & - & - & - & - & - & - & ta & ta & 0.014 & 0.036 \\
\hline (E)- $\beta$-santalol & 1732 & 1739 & - & - & - & - & - & - & - & - & 0.030 & 0.172 & 0.168 & 0.126 \\
\hline (Z)- $\alpha$-santalol acetate & 1780 & 1778 & - & - & - & - & - & - & - & - & 0.296 & 0.139 & 0.132 & 0.092 \\
\hline (Z)- $\alpha$-trans-bergamotol acetate & 1795 & 1794 & - & - & - & - & - & - & - & - & 0.207 & ta & ta & ta \\
\hline (Z)- $\beta$-santalol acetate & 1821 & 1819 & - & - & - & - & - & - & - & - & 0.012 & 0.017 & ta & ta \\
\hline hexahydrofarnesyl acetone & 1845 & 1844 & 0.075 & ta & ta & ta & 0.009 & ta & ta & ta & 0.014 & ta & ta & ta \\
\hline isopimara-9(11),15-diene & 1946 & 1905 & 0.028 & 0.014 & ta & ta & 0.019 & 0.014 & ta & ta & ta & 0.008 & ta & ta \\
\hline isopimara-8,15-diene & 1956 & 1947 & 0.032 & 0.017 & ta & ta & 0.022 & 0.014 & ta & ta & 0.020 & 0.007 & ta & ta \\
\hline sandaracopimara-8(14),15-diene & 1963 & 1969 & 0.075 & ta & ta & ta & 0.014 & ta & ta & ta & 0.021 & ta & ta & ta \\
\hline Monoterpene hydrocarbons & & & 44.129 & 46.541 & 45.223 & 63.426 & 47.939 & 44.206 & 48.712 & 52.070 & 44.717 & 44.637 & 49.040 & 61.576 \\
\hline Oxygenated monoterpenes & & & 2.561 & 1.681 & 1.382 & 1.611 & 2.918 & 1.315 & 1.141 & 1.967 & 1.319 & 0.937 & 0.964 & 0.600 \\
\hline Sesquiterpene hydrocarbons & & & 39.683 & 40.834 & 46.600 & 31.881 & 41.388 & 44.908 & 46.209 & 43.322 & 35.460 & 37.470 & 33.926 & 26.870 \\
\hline Oxygenated sesquiterpenes & & & 8.284 & 3.208 & 5.262 & 2.527 & 5.137 & 1.445 & 2.781 & 1.282 & 14.680 & 11.068 & 13.364 & 7.117 \\
\hline Others $\mathrm{F}$ & & & 2.271 & 0.676 & 0.837 & 0.532 & 1.996 & 0.847 & 1.124 & 0.855 & 1.281 & 0.916 & 0.993 & 0.607 \\
\hline Total identified & & & 96.93 & 92.94 & 99.30 & 99.98 & 99.38 & 92.72 & 99.97 & 99.50 & 97.46 & 95.03 & 98.29 & 96.77 \\
\hline
\end{tabular}


The substance $\alpha$-humulene is the chemical marker in the pharmaceutical industry for the quality control of V. curassavica essential oil, which varied from $1.47 \%$ to $3.93 \%$. Variance analysis (ANOVA) demonstrated significant differences $(p<0.05)$ between the seasons and between the genotypes evaluated (Table 3). Spring and summer were the seasons with the highest average production of the active principle $\alpha$-humulene (3.82\% and 3.93\%, respectively), while autumn was the season with the lowest average production (1.38\%). The highest average relative proportion was observed in the VC2 genotype during summer $(3.93 \pm 0.10)$ (Table 3$)$. Besides environmental factors, genetic characteristics can also determine and modify the production rate of essential oils [58-60].

Table 3. Contents of $\alpha$-humulene (\% Relative and SE Average) in the EO obtained from V. curassavica genotypes harvested at different seasons and analyzed by GC $\times$ GC-MS. Distinct uppercase letters $(\mathrm{A}, \mathrm{B}$ and $\mathrm{AB})$ for the rows and lowercase $(\mathrm{a}, \mathrm{b}$ and $\mathrm{ab})$ for columns represent statistically significant differences $(p<0.05)$.

\begin{tabular}{ccccc}
\hline \multirow{2}{*}{ Genotype } & \multicolumn{4}{c}{$\alpha$-Humulene } \\
\cline { 2 - 5 } & Winter $^{A B}$ & Spring $^{A}$ & Summer $^{A}$ & Autumn $^{\text {B }}$ \\
\hline VC1 $^{\text {b }}$ & $1.47 \pm 0.45$ & $2.38 \pm 0.39$ & $2.44 \pm 0.19$ & $1.38 \pm 0.49$ \\
VC2 $^{\text {a }}$ & $2.65 \pm 0.06$ & $3.82 \pm 0.31$ & $3.93 \pm 0.10$ & $3.28 \pm 0.21$ \\
VC3 $^{\text {ab }}$ & $2.89 \pm 0.14$ & $3.46 \pm 0.09$ & $2.88 \pm 0.16$ & $1.57 \pm 0.42$ \\
\hline
\end{tabular}

The heatmap plot (Figure 4), generated from hierarchical clustering, provides an overview of $V$. curassavica metabolic profile in response to the seasonal cycle and plant genetics. It is possible to observe that the genotype was responsible for most of the variance observed in the samples, highlighting two main blocks. The first block (upper eighteen rows) comprises a group of metabolites that are most abundant and present/absent in the VC3 genotype. For instance, $\alpha$-pinene (2) is most abundant in the samples of VC3 genotype. The (E)- $\alpha$-bergamotenal (53), (E)- $\alpha$-santalal (54), e $\alpha$-santalene (27) are present only in the VC3 genotype. The second block showed similar metabolic profiles, which is the case for the VC1 and VC2 genotypes, which is ascertained by the results attained with PCA analysis, as presented in the following paragraphs.

The most different chemical composition was found for the VC3 genotype (Figure 4), suggesting the contribution of other regulatory levels, besides environmental and genetic, in the chemical composition of the essential oils, e.g., physiological cycle of the plant and interaction between genotype and environment. The observations on the intra-class variance may contribute to V. curassavica breeding programs aiming at increasing the production of substances with biological activity.

To investigate the effects of seasonality on the volatile compounds of $V$. curassavica, a peak table-based multivariate approach, namely, principal component (PCA) was adopted for pattern recognition of the three evaluated genotypes. A two-component PCA model expressed $71.8 \%$ of the total variance with PC1 being responsible for describing $61.8 \%$ and PC2 $10 \%$ of the variance. The scores graph illustrated in Figure 5A indicated that the information contained in PC1 may be used to distinguish the samples into two groups (VC3 and VC1 + VC2). The loadings plot (Figure 5B) describes the most important variables that contributed to such pattern.

The substances responsible for the observed clustering between the samples were $\alpha$-pinene (2), found in a higher relative proportion in the VC3 genotype samples, and (E)- $\alpha$-bisabolene (42), (E)- $\beta$-santalol (56), (E)- $\alpha$-bergamotenal (53), (E)- $\alpha$-santalal (54), $\alpha$-santaleno (27), cis- $\alpha$-bergamotene (26), (Z)- $\beta$-Santalol acetate (59), (E)- $\alpha$-bergamotene (29), (Z)- $\beta$-farnesene (31), (E)- $\beta$-farnesene (33), (E)- $\gamma$-bisabolene (40), (Z)- $\beta$-Santalol (55), (Z)-caryophyllene (25), (Z)- $\alpha$-Santalol acetate (57), (Z)- $\alpha$-trans-bergamotol (52), and (Z)- $\alpha$-trans-bergamotol acetate (58). 


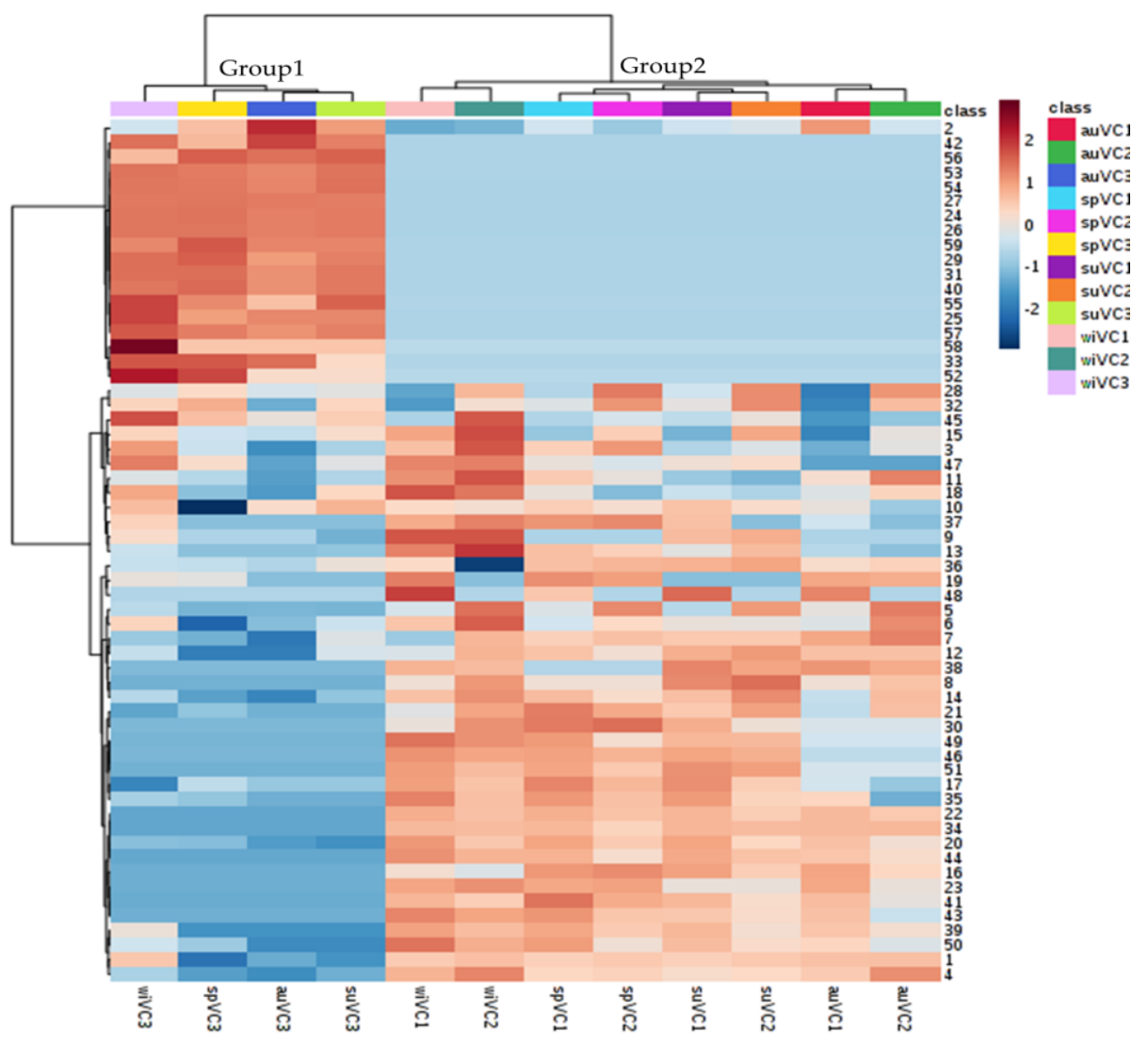

Figure 4. Heatmap generated from hierarchical clustering of the chemical composition of the essential oils of the three genotypes of $V$. curassavica as a function of the seasons. (Distance measure using Euclidean, and clustering algorithm using Ward's method).

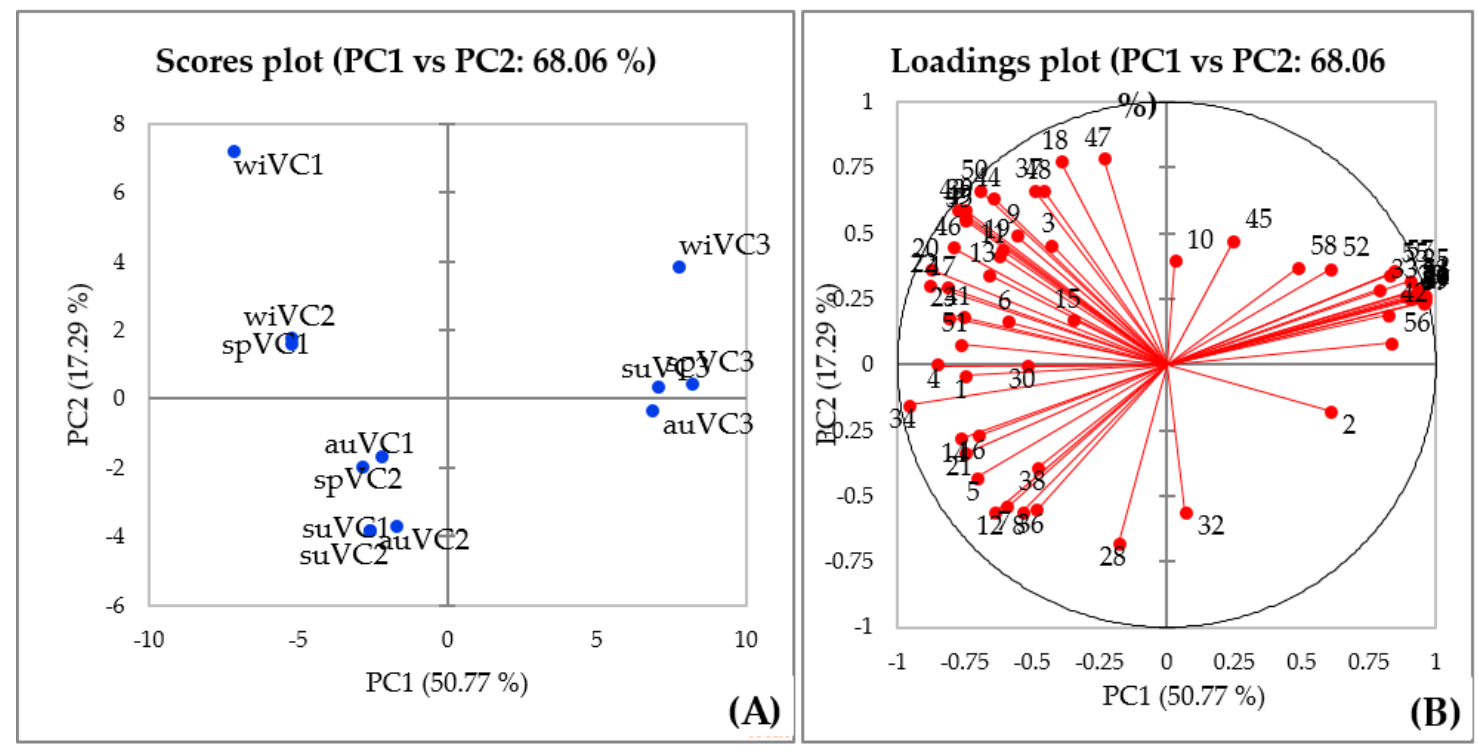

Figure 5. Scores plot (A) and loadings plot (B) obtained from PCA of data on the chemical composition of the V. curassavica essential oil.

In the current study, our results suggested that the genetic make-up is likely the most important factor contributing to the chemical composition of $V$. curassavica essential oils, whereas seasonal contributes to modulate the metabolite profile. Several factors have been demonstrated to contribute 
to the metabolic profile of plant essential oils [61,62], such as abiotic stress [63-65], genetics [58,59], and plant-pathogen interactions [60,66-68]. Furthermore, environmental biotic and abiotic conditions modulate several regulatory levels determined by the plant genotype affecting the profile of specialized metabolites of essential oils $[57,69]$. The determination of the contribution of distinct factors to the chemical profile of plant essential oils may help breeding and agricultural strategies to increase the yield of metabolites of interest, as well as in the selection of genotypes for the development of breeding programs.

\section{Conclusions}

Two-dimensional gas chromatography has proved to be a versatile tool for analyzing complex mixtures such as V. curassavica essential oil. GC $\times$ GC-QMS allowed the identification of 135 constituents in the essential oil, three times more when compared to 1D-GC. The improved resolving power of GC $\times$ GC has resolved co-eluting peaks facilitating the detection of trace substances in the essential oil of $V$. curassavica. The metabolic study of volatile constituents showed that the metabolic profile and the content of the anti-inflammatory active principle, $\alpha$-humulene, were significantly influenced by the genotype and seasonality. The content of the active principle was higher during spring and summer, being recommended for leaf harvest. Among the investigated genotypes, VC2 displayed higher amounts of the active principle.

Author Contributions: R.F., M.O.M.M. and L.W.H. conceived and planned the experiments. R.F. performed the experiments and collected and processed all the data. R.F. and L.W.H. wrote the paper with input from all authors. All authors have read and agreed to the published version of the manuscript.

Funding: This research was funded by National Council for Scientific and Technological Development (CNPq grant N. 400182/2016-5, fellowships 164918/2017-5 and 302748/2018-0) and the São Paulo Research Foundation (FAPESP, Grant N. 17/25490-1).

Acknowledgments: Nova Analítica and ThermoFisher Scientific are also acknowledged for supporting our research.

Conflicts of Interest: The authors declare no conflicts of interest.

\section{References}

1. Gilbert, B.; Favoreto, R. Cordia verbenacea DC. Boraginaceae. Revista Fitos 2012, 7, 17-25.

2. Aché Laboratórios Farmacêuticos Brazil. Available online: http://www.ache.com.br/arquivos/2605HellevachegaaoMéxicoeoAcheflantambem.pdf (accessed on 8 January 2020).

3. Oliveira, M. Laboratório em renovação. Pesquisa Fapesp 2017, 255, 74-77.

4. Fernandes, E.S.; Passos, G.F.; Medeiros, R.; Cunha, F.M.; Ferreira, J.; Campos, M.M.; Pianowski, L.F.; Calixto, J.B. Anti-inflammatory effects of compounds alpha-humulene and (-)-trans-caryophyllene isolated from the essential oil of Cordia verbenacea. Eur. J. Pharmacol. 2007, 569, 228-236. [CrossRef] [PubMed]

5. Passos, G.F.; Fernandes, E.S.; Cunha, F.M.; Ferreira, J.; Pianowski, L.F.; Campos, M.M.; Calixto, J.B. Anti-inflammatory and anti-allergic properties of the essential oil and active compounds from Cordia verbenacea. J. Ethnopharmacol. 2007, 110, 323-333. [CrossRef] [PubMed]

6. Medeiros, R.; Passos, G.F.; Vitor, C.E.; Koepp, J.; Mazzuco, T.L.; Pianowski, L.F.; Campos, M.M.; Calixto, J.B. Effect of two active compounds obtained from the essential oil of Cordia verbenacea on the acute inflammatory responses elicited by LPS in the rat paw. Br. J. Pharmacol. 2007, 151, 618-627. [CrossRef] [PubMed]

7. Roldão, E.F.; Witaicenis, A.; Seito, L.N.; Hiruma-Lima, C.A.; Di Stasi, L.C. Evaluation of the antiulcerogenic and analgesic activities of Cordia verbenacea DC. (Boraginaceae). J. Ethnopharmacol. 2008, 119, 94-98. [CrossRef]

8. Oliveira, D.M.; Luchini, A.C.; Seito, L.N.; Gomes, J.C.; Crespo-López, M.E.; Di Stasi, L.C. Cordia verbenacea and secretion of mast cells in different animal species. J. Ethnopharmacol. 2011, 135, 463-468. [CrossRef]

9. Michielin, E.M.Z.; Wiese, L.P.L.; Ferreira, E.A.; Pedrosa, R.C.; Ferreira, S.R.S. Radical-scavenging activity of extracts from Cordia verbenacea DC obtained by different methods. J. Supercrit. Fluids 2011, 56, 89-96. [CrossRef] 
10. Parisotto, E.B.; Michielin, E.M.Z.; Biscaro, F.; Ferreira, S.R.S.; Filho, D.W.; Pedrosa, R.C. The antitumor activity of extracts from Cordia verbenacea D.C. obtained by supercritical fluid extraction. J. Supercrit. Fluids 2012, 61, 101-107. [CrossRef]

11. Pimentel, S.P.; Barrella, G.E.; Casarin, R.C.V.; Cirano, F.R.; Casati, M.Z.; Foglio, M.A.; Figueira, G.F.; Ribeiro, F.V.R. Protective effect of topical Cordia verbenacea in a rat periodontitis model: Immune-inflammatory, antibacterial andmorphometric assays. BMC Complement. Altern. Med. 2012, 12, 224. [CrossRef]

12. Rodrigues, F.F.; Oliveira, L.G.; Rodrigues, F.F.; Saraiva, M.E.; Almeida, S.C.; Cabral, M.E.; Campos, A.R.; Costa, J.G. Chemical composition, antibacterial and antifungal activities of essential oil from Cordia verbenacea DC leaves. Pharmacogn. Res. 2012, 4, 161-165. [CrossRef] [PubMed]

13. Matias, E.F.F.; Alves, E.F.; Silva, M.K.N.; Carvalho, V.R.A.; Medeiros, C.R.; Santos, F.A.V.; Bitu, V.C.N.; Souza, C.E.S.; Figueredo, F.G.; Boligon, A.A.; et al. Potentiation of antibiotic activity of aminoglycosides by natural products from Cordia verbenacea DC. Microb. Pathog. 2016, 95, 111-116. [CrossRef] [PubMed]

14. Martins, E.R.; Díaz, I.E.C.; Paciencia, M.L.B.; Fana, S.A.; Morais, D.; Eberlin, M.N.; Silva, J.S.; Silveira, E.R.; Barros, M.P.; Suffredini, I.B. Interference of seasonal variation on the antimicrobial and cytotoxic activities of the essential oils from the leaves of Iryanthera polyneura in the Amazon Rain Forest. Chem. Biodivers. 2019, 16, e1900374. [CrossRef] [PubMed]

15. Zouari-Bouassida, K.; Trigui, M.; Makni, S.; Jlatel, L.; Tounsi, S. Seasonal variation in essential oils composition and the biological and pharmaceutical protective effects of Mentha longifolia leaves grown in Tunisia. BioMed. Res. Int. 2018, e7856517. [CrossRef]

16. Ferraz, E.O.; Vieira, M.A.R.; Ferreira, M.I.; Fernandes, A., Jr.; Marques, M.O.M.; Minatel, I.O.; Albano, M.; Sambo, P.; Lima, G.P.P. Seasonality effects on chemical composition, antibacterial activity and essential oil yield of three species of Nectandra. PLoS ONE 2018, 13, e0204132. [CrossRef]

17. Sun, C.X.; Li, M.Q.; Gao, X.X.; Liu, L.N.; Wu, X.F.; Zhou, J.H. Metabolic response of maize plants to multi-factorial abiotic stress. Plant Biol. 2016, 18, 120-129. [CrossRef] [PubMed]

18. Asghari, G.; Gholamali, H.; Mahmoudi, Z.; Asghari, M. Diurnal variation of essential of the oil components of Pycnocycla spinosa Decne. ex Boiss. Jundishapur. J. Nat. Pharm. Prod. 2014, 9, 35-38. [CrossRef]

19. Gonçalves, E.; Figueiredo, A.C.; Barroso, J.G.; Henriques, J.; Sousa, E.; Bonifácio, L. Effect of Monochamus galloprovincialis feeding on Pinus pinaster and Pinus pinea, oleoresin and insect valatiles. Phytochemistry 2020, 169, 112159. [CrossRef]

20. Medbouhi, A.; Benbelaïd, F.; Djabou, N.; Beaufay, C.; Bendahou, M.; Quetin-Leclercq, J.; Tintaru, A.; Costa, J.; Muselli, A. Essential Oil of Algerian Eryngium campestre: Chemical Variability and Evaluation of Biological Activities. Molecules 2019, 24, 2575. [CrossRef]

21. Dosoky, N.S.; Satyal, P.; Barata, L.M.; Silva, J.K.R.; Setzer, W.N. Volatiles of Black Pepper Fruits (Piper nigrum L.). Molecules 2019, 24, 4244. [CrossRef]

22. Souza, T.S.; Da Silva Ferreira, M.F.; Menini, L.; De Lima Souza, J.R.C.; De Oliveira Bernardes, C.; Ferreira, A. Chemotype diversity of Psidium guajava L. Phytochemistry 2018, 153, 129-137. [CrossRef] [PubMed]

23. Roberts, M.T.; Dufour, J.P.; Lewis, A.C. Application of comprehensive two-dimensional gas chromatography combined with time of flight mass spectrometry (GCxGC-TOFMS) for high resolution analysis of hop essential oil. J. Sep. Sci. 2004, 27, 473-478. [CrossRef] [PubMed]

24. Santos, T.G.; Fukuda, K.; Kato, M.J.; Sartorato, A.; Duarte, M.C.T.; Ruiz, A.L.T.G.; Carvalho, J.E.; Augusto, F. Characterization of the essential oils of two species of Piperaceae by one- and two-dimensional chromatographic techniques with quadrupole mass spectrometric detection. Microchem. J. 2014, 115, 113-120. [CrossRef]

25. Koek, M.M.; Kloet, F.M.; Kleemann, R.; Kooistra, T.; Verheij, E.R.; Hankemeier, T. Semi-automated non-target processing in GC $\times$ GC-MS metabolomics analysis: Applicability for biomedical studies. Metabolomics 2011, 7, 1-14. [CrossRef]

26. Dallüge, J.; Beens, J.; Brinkman, U.A.T. Comprehensive two-dimensional gas chromatography: A powerful and versatile analytical tool. J. Chromatogr. A 2003, 1000, 69-108. [CrossRef]

27. Tranchida, P.Q.; Franchina, F.A.; Mondello, L. Analysis of essential oils through comprehensive two-dimensional gas chromatography: General utility. Flavour Frag. J. 2017, 32, 218. [CrossRef]

28. Wong, Y.F.; Perlmutter, P.; Marriott, P.J. Untargeted metabolic profiling of Eucalyptus spp. leaf oils using comprehensive two-dimensional gas chromatography with high resolution mass spectrometry: Expanding the metabolic coverage. Metabolomics 2017, 13, 46. [CrossRef] 
29. Seeley, J.V.; Seeley, S.K. Multidimensional gas chromatography: Fundamental advances and new applications. Anal. Chem. 2013, 85, 557-578. [CrossRef]

30. Meinert, C.; Meierhenrich, U.J. A new dimension in separation science: Comprehensive two-dimensional gas chromatography. Angew. Chem. Int. Ed. Engl. 2012, 51, 10460-10470. [CrossRef]

31. Pedroso, M.P.; Godoy, L.A.F.; Fidélis, C.H.V.; Ferreira, E.C.; Poppi, R.J.; Augusto, F. Cromatografia gasosa bidimensional abrangente (GC×GC). Quím. Nova 2009, 32, 421-430. [CrossRef]

32. Duhamel, C.; Cardinael, P.; Peulon-agasse, V.; Firor, R.; Pascaud, L.; Semard-jousset, G.; Giusti, P.; Livadaris, V. Comparison of cryogenic and differential flow (forward and reverse fill/flush) modulators and applications to the analysis of heavy petroleum cuts by high-temperature comprehensive gas. J. Chromatogr. A 2015, 1387, 95-103. [CrossRef] [PubMed]

33. Cordero, C.; Rubiolo, P.; Reichenbach, S.E.; Carreta, A.; Cobelli, L.; Giardina, M.; Bicchi, C. Method translation and full metadata transfer from thermal to differential flow modulated comprehensive two dimensional gas chromatography: Profiling of suspected fragrance allergens. J. Chromatogr. A 2017, 1480, 70-82. [CrossRef] [PubMed]

34. Cordero, C.; Rubiolo, P.; Cobelli, L.; Stani, G.; Miliazza, A.; Giardina, M.; Firor, R.; Bicchi, C. Potential of the reversed-inject dofferential flow modulator for comprehensive two-dimensional gas chromatography in the quantitative profiling and fingerprinting of essenctial oils of different complexity. J. Chromatogr. A 2015, 1417, 79-95. [CrossRef] [PubMed]

35. Barbará, J.A.; Nicolli, K.P.; Souza-Silva, E.A.; Biasoto, A.C.T.; Welke, J.E.; Zini, C.A. Volatile profile and aroma potential of tropical Syrah wines elaborated in different maturation and maceration times using comprehensive two-dimensional gas chromatography and olfactometry. Food Chem. 2020, 308, 125552. [CrossRef]

36. Lukić, I.; Carlin, S.; Horvat, I.; Vrhovsek, U. Combined targeted and untargeted profiling of volatile aroma compounds with comprehensive two-dimensional gas chromatography for differentiation of virgin olive oils according to variety and geographical. Food Chem. 2019, 270, 403-414. [CrossRef] [PubMed]

37. Dos Santos, A.L.; Novaes, A.D.S.; Polidoro, A.D.S.; Barros, M.E.; Mota, J.S.; Lima, D.B.M.; Krause, L.C.; Cardosos, C.A.L.; Jacques, R.A.; Camarão, E.B. Chemical characterisation of Piper amalogo (Piperaceae) essential oil by comprehensive two-dimensional gas chromatography coupled with rapid-scanning quadrupole mass spectrometry (GC $\times \mathrm{GC} / \mathrm{qMS})$ and their antilithiasic activity and acute toxicity. Phytochem. Anal. 2018, 29, 432-445. [CrossRef]

38. Yan, D.; Wong, Y.F.; Tedone, L.; Shellie, R.A.; Marriott, P.J.; Whittock, S.P.; Koutoulis, A. Chemotyping of new hop (Humulus lupulus L.) genotypes using comprehensive two-dimensional gas chromatography with quadrupole accurate mass-time-of-flight mass spectrometry. J. Chromatogr. A 2018, 1536, 110-121. [CrossRef]

39. Filippi, J.J.; Belhassen, E.; Baldovini, N.; Brevard, H.; Meierhenrich, U.J. Qualitative and quantitative analysis of vetiver essential oils by comprehensive two-dimensional gas chromatography and comprehensive two-dimensional gas chromatography/mass spectrometry. J. Chromatogr. A 2013, 1288, 127-148. [CrossRef]

40. Fidelis, C.H.V.; Augusto, F.; Sampaio, P.T.B.; Krainovic, P.M.; Barata, L.E.S. Chemical characterization of rosewood (Aniba rosaeodora Ducke) leaf essential oil by comprehensive two-dimensional gas chromatography coupled with quadrupole mass spectrometry. J. Essent. Oil Res. 2012, 24, 245-251. [CrossRef]

41. Keppler, E.A.H.; Jenkins, C.L.; Davis, T.J.; Bean, H.D. Advances in the application of comprehensive two-dimensional gas chromatography in metabolomics. TrAC Trend Anal. Chem. 2018, 109, 275-286. [CrossRef]

42. Morimoto, J.; Rosso, M.C.; Kfoury, N.; Bicchi, C.; Cordero, C.; Robbat, A., Jr. Untargeted/Targeted 2D gas chromatography/mass spectrometry detection of the total volatile Tea metabolome. Molecules 2019, 24, 3757. [CrossRef] [PubMed]

43. Da Ros, A.; Masuero, D.; Riccadonna, S.; Bubola, K.B.; Mulinacci, N.; Mattivi, F.; Lukić, I.; Vrhovsek, U. Complementary untargeted and targeted metabolomics for differentiation of extra virgin Olive oils of different origin of purchase based on volatile and phenolic composition and sensory quality. Molecules 2019, 24, 2896. [CrossRef] [PubMed]

44. Sciarrone, D.; Giuffrida, D.; Rotondo, A.; Micalizzi, G.; Zoccali, M.; Pantò, S.; Donato, P.; Rodrigues-das-Dores, R.G.; Mondello, L. Quali-quantitative characterization of the volatile constituints in Cordia verbenacea D.C. essential oil exploiting advanced chromatographic approaches and nuclear magnetic resonance analysis. J. Chromatogr. A 2017, 1524, 246-253. [CrossRef] [PubMed] 
45. Silva, K.P.; Santos, T.A.C.; Moutinho, B.L.; Silva, R.S.; Pinto, V.S.; Blank, A.F.; Corrêa, C.B.; Scher, R.; Fernandes, R.P.M. Using Varronia curassavica (Cordiaceae) essential oil for the biocontrolo f Phytomonas serpens. Ind. Crops Prod. 2019, 139, 111523. [CrossRef]

46. Matias, E.F.F.; Alves, E.F.; Silva, M.K.N.; Carvalho, V.R.A.; Fegueiredo, F.G.; Ferreira, J.V.A.; Coutinho, H.D.M.; Silva, M.F.L.; Ribeiro-Filho, J.; Costa, J.G.M. Seasonal variation, chemical composition and biological activity of theessential oil of Cordia verbenacea DC (Boraginaceae) and the sabinene. Ind. Crops Prod. 2016, 87, 45-53. [CrossRef]

47. Queiroz, T.B.; Mendes, A.D.R.; Silva, J.C.R.L.; Fonseca, F.S.A.; Martins, E.R. Content and chemical composition of the essential oil of 'erva-baleeira' (Varronia curassavica Jaqc.) as a function of harvesting times. Rev. Bras. Plantas Med. 2016, 18, 356-362. [CrossRef]

48. Marques, A.P.S.; Bonfim, F.P.G.; Dantas, W.F.C.; Puppi, R.J.; Marques, M.O.M. Chemical composition of essential oil from Varronia curassavica Jacq. accessions in different seasons of the year. Ind. Crops Prod. 2019, 140, 111656. [CrossRef]

49. Santos, A.V.; Defaveri, A.C.A.; Bizzo, H.R.; Gil, R.A.S.S.; Sato, A. In vitro propagation, histochemistry, and analysis of essential oil from conventionally propagated and in vitro-propagated plants of Varronia curassavica Jacq. In Vitro Cell. Dev. Biol. Plant 2013, 49, 405-413. [CrossRef]

50. CIIAGRO - Centro Integrado de Informações Agrometeorológicas. Instituto Agronômico de Campinas (IAC). Available online: http://www.ciiagro.sp.gov.br/ (accessed on 8 January 2020).

51. Adams, R.P. Identification of Essential Oil Components by Gas. Cromatography/Mass Spectroscopy, 4.1rd ed.; Allured Publ. Corp: Carol Stream, IL, USA, 2017.

52. Van Den Dool, H.; Kratz, D.J. A generalization of the retention index system incluing liner temperature programmed gas-liquid partition chromatograpy. J. Cromatogr. 1963, 11, 463-467. [CrossRef]

53. Chong, J.; Wishart, D.S.; Xia, J. Using MetaboAnalyst 4.0 for comprehensive and integrative metabolomics data analysis. Curr. Protoc. Bioinforma. 2019, 68, e86. [CrossRef]

54. Fidelis, C.H.V.; Sampaio, P.T.B.; Krainovic, P.M.; Augusto, F.; Barata, L.E.S. Correlation between maturity of tree and GCxGC-qMS chemical profiles of essential oil from leaves of Aniba rosaeodora Ducke. Microchem. J. 2013, 109, 73-77. [CrossRef]

55. Kaya, D.A.; Arslan, M.; Rusu, L.-C. Effects of harvesting hour on essential oil content and composition of Thymus vulgaris. Farmacia 2013, 61, 1194-1203.

56. Lakušić, B.S.; Ristić, M.S.; Slavkovska, V.N.; Stojanović, D.L.; Lakušić, D.V. Variations in essential oil yields and compositions of Salvia officinalis (Lamiaceae) at different developmental stages. Botanica Serbica 2013, 37, 127-139.

57. Mishra, R.; Gupta, A.K.; Kumar, A.; Lal, R.K.; Saikia, D.; Chanotiya, C.S. Genetic diversity, essential oil composition, and in vitro antioxidant and antimicrobial activity of Curcuma longa L. germplasm collections. J. Appl. Res. Med. Aromat. Plants 2018, 10, 75-84. [CrossRef]

58. Alemu, A.; Garedew, W.; Gebre, A. Essential oil yield and yield components of basil (Ocimum basilicum L.) as affected by genotype and intrarow spacing at Jimma, SW Ethiopia. Acta Agrobot. 2018, 71, 1743. [CrossRef]

59. Külheim, C.; Padovan, A.; Hefer, C.; Krause, S.T.; Köllner, T.G.; Myburg, A.A.; Degenhardt, J.; Foley, W.F. The Eucalyptus terpene synthase gene family. BMC Genom. 2015, 16, 450. [CrossRef]

60. Facanali, R.; Colombo, C.A.; Teixeira, J.P.F.; Ming, L.C.; Zucchi, M.I.; Marques, M.O.M. Genetic and chemical diversity of native populations of Ocimum selloi Benth. Ind. Crops Prod. 2015, 76, 249-257. [CrossRef]

61. Padovan, A.; Keszei, A.; Külheim, C.; Foley, W.J. The evolution of foliar terpene diversity in Myrtaceae. Phytochem. Rev. 2014, 13, 695-716. [CrossRef]

62. Souza, T.S.; Ferreira, M.F.S.; Menini, L.; Souza, J.R.C.L.; Parreira, L.A.; Cecon, P.R.; Ferreira, A. Essential oil of Psidium guajava: Influence of genotypes and environment. Sci. Hortic. 2017, 216, 38-44. [CrossRef]

63. Gasparetto, A.; Cruz, A.B.; Wagner, T.M.; Bonomini, T.J.; Correa, R.; Malheiros, A. Seasonal variation in the chemical composition, antimicrobial and mutagenic potential of essential oils from Piper cernuum. Ind. Crops Prod. 2017, 95, 256-263. [CrossRef]

64. Macêdo, D.G.; Souza, M.M.A.; Morais-Braga, M.F.B.; Coutinho, H.D.M.; Santos, A.T.L.; Cruz, R.P.; Costa, J.G.M.; Rodrigues, F.F.G.; Quintans-junior, L.J.; Almeida, J.R.G.S.; et al. Effect of seasonality on chemical profile and antifungal activity of essential oil isolated from leaves Psidium salutare (Kunth) O. Berg. PeerJ 2018, 6, e5476. [CrossRef] [PubMed] 
65. Gomes, A.F.; Almeida, M.P.; Leite, M.F.; Schwaiger, S.; Stuppner, H.; Halabalaki, M.; Amaral, J.G.; David, J.M. Seasonal variation in the chemical composition of two chemotypes of Lippia alba. Food Chem. 2019, 273, 186-193. [CrossRef] [PubMed]

66. Pinto, J.A.O.; Blank, A.F.; Nogueira, P.C.L.; Arrigoni-Blank, M.F.; Andrade, T.M.; Sampaio, T.S.; Pereira, K.L.G. Chemical chracterization of the essential oil from leaves of basil genotypes cultivated in different seasons. Bol. Latinoam. Caribe Plant Med. Aromat. 2019, 18, 58-70. [CrossRef]

67. Mendes, L.A.; Souza, T.S.; Menini, L.; Guilhen, J.H.S.; Bernardes, C.O.; Ferreira, A.; Ferreira, M.F.S. Spring alterations in the chromatographic profile of leaf essential oils of improved guava genotypes in Brazil. Sci. Hortic. 2018, 238, 295-302. [CrossRef]

68. He, X.; Wang, S.; Shi, J.; Sun, Z.; Lei, Z.; Yin, Z.; Qian, Z.; Tang, H.; Xie, H. Genotypic and Environmental Effects on the Volatile Chemotype of Valeriana jatamansi Jones. Front. Plant Sci. 2018, 9, 1003. [CrossRef]

69. Schluttenhofer, C.; Yuan, L. Regulation of specialized metabolism by WRKY transcription factors. Plant Physiol. 2015, 167, 295-306. [CrossRef]

(C) 2020 by the authors. Licensee MDPI, Basel, Switzerland. This article is an open access article distributed under the terms and conditions of the Creative Commons Attribution (CC BY) license (http://creativecommons.org/licenses/by/4.0/). 